\title{
ON APPROXIMATE ANTIGRADIENTS
}

\author{
XIAO-XIONG GAN AND KARL R. STROMBERG
}

(Communicated by Andrew M. Bruckner)

\begin{abstract}
For $n \in \mathbb{N}$ and $I=[0,1]$, let $I^{n}$ be the unit cube and $\lambda^{n}$ the Lebesgue measure in $\mathbb{R}^{n}$. It is proved that if $f: I^{n} \rightarrow \mathbb{R}^{n}$ and $F_{0}: I^{n} \rightarrow \mathbb{R}$ are continuous and $\varepsilon>0$, then there exist a continuous $F: I^{n} \rightarrow \mathbb{R}$ and an open set $W \subset\left(I^{n}\right)^{\circ}$ with $\lambda^{n}(W)=1$ such that

(i) $\nabla F$ exists and is continuous on $W$,

(ii) $\|\nabla F(x)-f(x)\|<\varepsilon \quad \forall x \in W$, and

(iii) $\left|F(x)-F_{0}(x)\right|<\varepsilon \quad \forall x \in I^{n}$, where $\|y\|=\left(\sum_{j=1}^{n} y_{j}^{2}\right)^{1 / 2} \quad \forall y \in \mathbb{R}^{n}$.
\end{abstract}

\section{INTRODUCTION AND DEFINITIONS}

If $f \in C([0,1])$ and $F(x)=\int_{0}^{x} f$ for $0 \leq x \leq 1$, then $F^{\prime}=f$. A famous theorem of Lusin states that if $\phi: I \rightarrow \mathbb{R}$ is a measurable function, then there exists $F \in C([0,1])$ such that $F^{\prime}=\phi$ a.e. on $[0,1]$ (see [1, p. 217]). However, if $f(x, y)=(0, x)$ for $(x, y) \in I^{2}$, then there is no $F: I^{2} \rightarrow \mathbb{R}$ for which $\nabla F=f$, because $D_{1} F=0$ implies that $F$ depends only on $y$. Thus, if $n>1$ and $f \in C\left(I^{n}, \mathbb{R}^{n}\right)$, then $f$ need not have an antigradient $F: \nabla F=f$. Can we find some kind of approximate antigradient for $f$ ? More generally, if $f: I^{n} \rightarrow \mathbb{R}^{n}$ is only measurable, what can be said? We are going to give some answers to these questions.

Throughout this paper, we use some definitions listed below.

(0.1) Definitions. Let $m, n \in \mathbb{N}$ and a nonvoid set $\Omega$ be given.

(1) If $F: \Omega \rightarrow \mathbb{R}^{m}$ is a mapping, then for $1 \leq i \leq m$, define the $i$ th coordinate function $F_{i}: \Omega \rightarrow \mathbb{R}$ of $F$ by letting $F_{i}(t)$ be the $i$ th coordinate of $F(t)$ :

$$
F(t)=\left(F_{1}(t), F_{2}(t), \ldots, F_{m}(t)\right), \quad t \in \Omega .
$$

(2) If $\Omega$ is a topological space, then $C\left(\Omega, \mathbb{R}^{m}\right)$ denotes the family of all continuous mappings from $\Omega$ to $\mathbb{R}^{m}$. If $m=1$, write $C(\Omega)$ for $C(\Omega, \mathbb{R})$. Similarly, if $\Omega \subset \mathbb{R}^{n}$, we denote by $\mathfrak{M}\left(\Omega, \mathbb{R}^{m}\right)$ the set of all Lebesgue measurable mappings from $\Omega$ to $\mathbb{R}^{m}$ and write $\mathfrak{M}(\Omega)$ for $\mathfrak{M}(\Omega, \mathbb{R})$.

(3) Let $\Omega \subset \mathbb{R}^{n}$ and let $f \in C(\Omega)$. If $\frac{\partial f}{\partial x_{j}}(x)$ exists at some $x \in \Omega^{\circ}$ (the

Received by the editors March 30, 1992.

1991 Mathematics Subject Classification. Primary 26B35; Secondary 41A30, 35A35.

Key words and phrases. Antigradient. 
interior of $\Omega$ ) $\forall 1 \leq j \leq n$, we define

$$
\nabla f(x)=\left(\frac{\partial}{\partial x_{1}} f(x), \frac{\partial}{\partial x_{2}} f(x), \ldots, \frac{\partial}{\partial x_{n}} f(x)\right),
$$

and call $\nabla f$ the gradient of the function $f$. We also write $D_{j}$ for $\frac{\partial}{\partial x_{j}}$.

Conversely, let $f \in \mathfrak{M}\left(\Omega, \mathbb{R}^{n}\right)$. If there is $F \in C(\Omega)$ such that $\nabla F=f$, we call $F$ an antigradient of $f$.

(4) We always denote by $I^{n}$ the unit cube in $\mathbb{R}^{n}$. That is

$$
I^{n}=\underset{k=1}{n}[0,1]=\left\{x \in \mathbb{R}^{n}: 0 \leq x_{j} \leq 1, j=1,2, \ldots, n\right\} .
$$

Also, we always denote by $\lambda^{n}$ the Lebesgue measure on $\mathbb{R}^{n}$.

(5) Let $\Omega \subset \mathbb{R}^{n}$ and $\phi=\left(\phi_{1}, \phi_{2}, \ldots, \phi_{m}\right) \in \mathfrak{M}\left(\Omega, \mathbb{R}^{m}\right)$. If $\varepsilon>0$, then we say $\left\|\left(\phi_{1}, \phi_{2}, \ldots, \phi_{m}\right)\right\|<\varepsilon$ a.e. on $\Omega$ to mean that

$$
\lambda^{n}\left(\left\{x \in \Omega:\left\|\left(\phi_{1}(x), \phi_{2}(x), \ldots, \phi_{m}(x)\right)\right\| \geq \varepsilon\right\}\right)=0,
$$

where $\|y\|=\left(\sum_{i=1}^{m} y_{i}^{2}\right)^{1 / 2}$ if $y \in \mathbb{R}^{m}$.

(6) For any $F: \Omega \rightarrow \mathbb{R}$, we define the uniform norm of $F$ by

$$
\|F\|_{u}=\sup \{|F(x)|: x \in \Omega\} \text {. }
$$

(7) We reserve the letter $\psi$ to denote Lebesgue's singular function (see [2, p. 130] or $[4$, p. 113]). It is a continuous, nondecreasing function $\mathbb{R} \rightarrow[0,1]$ with $\psi(0)=0$ and $\psi(1)=1$ which is constant on each component interval of $\mathbb{R} \backslash C$, where $C$ is Cantor's ternary set.

\section{APPROXIMATION TO ANTIGRADIENTS}

(1.1) Theorem. Let $n \in \mathbb{N}$, let $P \in C\left(I^{n}, \mathbb{R}^{n}\right)$, and let $\varepsilon>0$. Then there exist a continuous $Q: I^{n} \rightarrow \mathbb{R}$ and an open $V \subset\left(I^{n}\right)^{\circ}$ with $\lambda^{n}(V)=1$ such that

(i) $\nabla Q$ exists and is continuous on $V$, and

(ii) $\|\nabla Q(x)-P(x)\|<\varepsilon \quad \forall x \in V$,

where $\|y\|=\left(\sum_{j=1}^{n} y_{j}^{2}\right)^{1 / 2} \quad \forall y \in \mathbb{R}^{n}$.

Proof. If $n=1$, define

$$
Q(x)=\int_{0}^{x} P(t) d t \quad(0 \leq x \leq 1)
$$

and take $V=] 0,1\left[=I^{\circ}\right.$. Then $\nabla Q=Q^{\prime}=P$ on $V$ so the theorem is true if $n=1$.

Suppose, as an induction hypothesis, that $n>1$ and that the theorem is true if $n$ is replaced with $n-1$. The uniform continuity of $P$ provides $\delta>0$ such that

$$
u, v \in I^{n},\|u-v\|<\delta \Rightarrow\|P(u)-P(v)\|<\varepsilon / 6 n .
$$

Choose $N \in \mathbb{N}$ with $N \delta>1$.

In this paragraph, let $k \in\{1,2, \ldots, n\}$ be given and fixed. We have $P(x)=$ $\left(P_{1}(x), P_{2}(x), \ldots, P_{n}(x)\right)$ for $x \in I^{n}$, where $P_{j} \in C\left(I^{n}\right)(1 \leq j \leq n)$. For $r \in\{0,1, \ldots, N\}$, we identify the slice

$$
S_{r}=\left\{x \in I^{n}: x_{k}=r / N\right\}
$$


with $I^{n-1}$ in the obvious way. Thus we write

$$
S_{r}^{\circ}=\left\{x \in S_{r}: 0<x_{j}<1 \forall j \neq k\right\} .
$$

For each such $r$, we apply our induction hypothesis to the restriction of $P$ to $S_{r}$ to obtain a continuous function $G_{r}: S_{r} \rightarrow \mathbb{R}$ and a set $V_{r} \subset S_{r}^{\circ}$ which is open in $S_{r}$ such that

$$
\begin{aligned}
& \lambda^{n-1}\left(V_{r}\right)=1, \\
& \frac{\partial G_{r}}{\partial x_{j}} \text { exists and is continuous on } V_{r} \forall j \neq k(1 \leq j \leq n) \text {, and } \\
& \left(\sum_{j=1, j \neq k}^{n}\left[\frac{\partial G_{r}}{\partial x_{j}}(x)-P_{j}(x)\right]^{2}\right)^{1 / 2}<\frac{\varepsilon}{6 n} \quad \forall x \in V_{r} .
\end{aligned}
$$

Next we construct a function $Q_{k}$ on $I^{n}$ which extends each $G_{r}$. For $x=$ $\left(x_{1}, x_{2}, \ldots, x_{n}\right) \in I^{n}$, define $x^{r} \in S_{r}$ by $\left(x^{r}\right)_{j}=x_{j}$ if $j \neq k$ and $\left(x^{r}\right)_{k}=r / N$. Let $\psi$ be Lebesgue's singular function in terms of Cantor's ternary set $C$ as in (0.1) (7), and define $f_{r}$ on the slab

$$
\left\{x \in I^{n}:(r-1) / N \leq x_{k} \leq r / N\right\}
$$

for $r=1,2, \ldots, N$ by the rule

$$
f_{r}(x)=G_{r-1}\left(x^{r-1}\right)+\left[G_{r}\left(x^{r}\right)-G_{r-1}\left(x^{r-1}\right)\right] \cdot \psi\left(N x_{k}-r+1\right) .
$$

Since $\psi(0)=0$ and $\psi(1)=1$, we have

$$
x \in S_{r-1} \Rightarrow x_{k}=(r-1) / N \Rightarrow x^{r-1}=x \Rightarrow f_{r}(x)=G_{r-1}(x) \text {, }
$$

and

$$
x \in S_{r} \Rightarrow x_{k}=r / N \Rightarrow x^{r}=x \Rightarrow f_{r}(x)=G_{r}(x) .
$$

Thus the formula

$$
Q_{k}(x)=f_{r}(x) \text { if }(r-1) / N \leq x_{k} \leq r / N
$$

unambiguously defines the function $Q_{k}: I^{n} \rightarrow \mathbb{R}$. The continuity of the functions $x \rightarrow x^{r}$, each $G_{r}$, and $\psi$ shows that $Q_{k}$ is continuous on $I^{n}$. Define

$$
\begin{aligned}
W_{k}= & \left(I^{n}\right)^{\circ} \cap\left(\bigcap_{r=0}^{N}\left\{x \in I^{n}: x^{r} \in V_{r}\right\}\right) \\
& \cap\left(\bigcup_{r=1}^{N}\left\{x \in I^{n}: r-1 \leq N x_{k} \leq r,\left(N x_{k}-r+1\right) \notin C\right\}\right),
\end{aligned}
$$

where $C$ is Cantor's ternary set. Then $W_{k}$ is an open subset of $\left(I^{n}\right)^{\circ}$ and $\lambda^{n}\left(W_{k}\right)=1$. By (3), (4), and the properties of the functions $G_{r}$, we see that for $j \neq k$ the partial derivative $\frac{\partial Q_{k}}{\partial x_{j}}$ exists and is continuous on $W_{k}$. Since $\psi^{\prime}=0$ on $[0,1] \backslash C$, we have

$$
\frac{\partial Q_{k}}{\partial x_{k}}(x)=0 \quad \forall x \in W_{k}
$$

Thus $\nabla Q_{k}$ is continuous on $W_{k}$. Now let $x \in W_{k}$ with $r-1<N x_{k}<r$. Then we have

$$
\left\|x-x^{r}\right\|<\left\|x^{r-1}-x^{r}\right\|=1 / N<\delta
$$


so we use (1)-(4) and the fact that $0 \leq \psi \leq 1$ to see that $j \neq k \quad(1 \leq j \leq n) \Rightarrow$

$$
\begin{aligned}
\left|\frac{\partial Q_{k}}{\partial x_{j}}(x)-\frac{\partial G_{r-1}}{\partial x_{j}}\left(x^{r-1}\right)\right|=\left|\frac{\partial f_{r}}{\partial x_{j}}(x)-\frac{\partial G_{r-1}}{\partial x_{j}}\left(x^{r-1}\right)\right| \\
=\left|\left[\frac{\partial G_{r}}{\partial x_{j}}\left(x^{r}\right)-\frac{\partial G_{r-1}}{\partial x_{j}}\left(x^{r-1}\right)\right] \cdot \psi\left(N x_{k}-r+1\right)\right| \\
\leq\left|\frac{\partial G_{r}}{\partial x_{j}}\left(x^{r}\right)-P_{j}\left(x^{r}\right)\right|+\left|P_{j}\left(x^{r}\right)-P_{j}\left(x^{r-1}\right)\right| \\
\quad+\left|P_{j}\left(x^{r-1}\right)-\frac{\partial G_{r-1}}{\partial x_{j}}\left(x^{r-1}\right)\right| \\
<\frac{\varepsilon}{6 n}+\frac{\varepsilon}{6 n}+\frac{\varepsilon}{6 n}=\frac{\varepsilon}{2 n},
\end{aligned}
$$

and

$$
\begin{aligned}
\left|\frac{\partial G_{r-1}}{\partial x_{j}}\left(x^{r-1}\right)-P_{j}(x)\right| & \leq\left|\frac{\partial G_{r-1}}{\partial x_{j}}\left(x^{r-1}\right)-P_{j}\left(x^{r-1}\right)\right|+\left|P_{j}\left(x^{r-1}\right)-P_{j}(x)\right| \\
& <\frac{\varepsilon}{6 n}+\frac{\varepsilon}{6 n}<\frac{\varepsilon}{2 n} .
\end{aligned}
$$

This shows that

$$
\left|\frac{\partial Q_{k}}{\partial x_{j}}(x)-P_{j}(x)\right|<\frac{\varepsilon}{n} \quad \text { if } x \in W_{k} \text { and } j \neq k(1 \leq j \leq n) .
$$

Define $P^{k}: I^{n} \rightarrow \mathbb{R}^{n}$ by

$$
P^{k}(x)=\left(P_{1}^{k}(x), P_{2}^{k}(x), \ldots, P_{n}^{k}(x)\right),
$$

where $P_{j}^{k}=P_{j}$ if $j \neq k$ and $P_{k}^{k}=0$. Then (5) and (6) yield that $x \in W_{k} \Rightarrow$

$$
\begin{aligned}
\left\|\nabla Q_{k}(x)-P^{k}(x)\right\|= & \left(\sum_{j=1, j \neq k}^{n}\left[\frac{\partial Q_{k}}{\partial x_{j}}(x)-P_{j}(x)\right]^{2}\right)^{1 / 2} \\
& \leq\left((n-1) \cdot\left(\frac{\varepsilon}{n}\right)^{2}\right)^{1 / 2} \leq \frac{n-1}{n} \varepsilon .
\end{aligned}
$$

Thus we have constructed $Q_{k}$ and $W_{k}$ for each $k \in\{1,2, \ldots, n\}$.

Finally, notice that $P=\frac{1}{n-1} \sum_{k=1}^{n} P^{k}$ and define

$$
V=\bigcap_{k=1}^{n} W_{k} \text { and } Q=\frac{1}{n-1} \sum_{k=1}^{n} Q_{k} .
$$

Then $V$ is an open subset of $\left(I^{n}\right)^{\circ}, \lambda^{n}(V)=1, Q \in C\left(I^{n}\right)$, and

(i) $\nabla Q$ is defined and continuous on $V$.

Also, (7) yields $x \in V \Rightarrow$

$$
\|\nabla Q(x)-P(x)\| \leq \frac{1}{n-1} \sum_{k=1}^{n}\left\|\nabla Q_{k}(x)-P^{k}(x)\right\|<\frac{1}{n-1} \sum_{k=1}^{n} \frac{n-1}{n} \varepsilon=\varepsilon .
$$

This is (ii). We have completed the proof. 
The next theorem tells us what can be done if the mapping $P$ in the above theorem is only measurable.

(1.2) Theorem. Let $n \in \mathbb{N}$ and let $\phi \in \mathfrak{M}\left(I^{n}, \mathbb{R}^{n}\right)$. Then there exists a sequence $\left\{Q_{k}\right\}_{k=1}^{\infty} \subset C\left(I^{n}\right)$ such that

(i) $\nabla Q_{k}$ exists and is continuous on some open set $V_{k} \subset\left(I^{n}\right)^{\circ}$ with $\lambda^{n}\left(V_{k}\right)=$ $1 \forall k \in \mathbb{N}$, and

(ii) $\lim _{k \rightarrow \infty} \nabla Q_{k}(x)=\phi(x)$ for almost every $x \in \bigcap_{k=1}^{\infty} V_{k}$.

Proof. Let $\phi=\left(\phi_{1}, \phi_{2}, \ldots, \phi_{n}\right)$ as we mentioned in $(0.1)$.

By Luzin's theorem, for each $k \in \mathbb{N}$ and $i \in\{1,2, \ldots, n\}$ there exist an open set $E_{k, i} \subset I^{n}$ with $\lambda^{n}\left(E_{k, i}\right)<2^{-k} / n$ and a function $f_{k, i} \in C\left(I^{n}\right)$ such that

$$
f_{k, i}(x)=\phi_{i}(x) \quad \forall x \in I^{n} \backslash E_{k, i} .
$$

Let $E_{k}=\bigcup_{i=1}^{n} E_{k, i}$. Then $\lambda^{n}\left(E_{k}\right)<2^{-k}$ and

$$
f_{k, i}(x)=\phi_{i}(x) \quad \forall x \in I^{n} \backslash E_{k}, \quad 1 \leq i \leq n .
$$

Take $f_{k}=\left(f_{k, 1}, f_{k, 2}, \ldots, f_{k, n}\right)$. Then $f_{k} \in C\left(I^{n}, \mathbb{R}^{n}\right)$ and

$$
f_{k}(x)=\phi(x) \quad \forall x \in I^{n} \backslash E_{k} \text { for each } k \in \mathbb{N} .
$$

Theorem (1.1) says that, for each $k \in \mathbb{N}$, there exist a $Q_{k} \in C\left(I^{n}\right)$ and an open set $V_{k} \subset\left(I^{n}\right)^{\circ}$ with $\lambda^{n}\left(V_{k}\right)=1$ such that $\nabla Q_{k}$ exists and is continuous on $V_{k}$ and

$$
\left\|\nabla Q_{k}(x)-f_{k}(x)\right\|<2^{-k} \quad \forall x \in V_{k} .
$$

Define $E=\bigcap_{r=1}^{\infty} \bigcup_{k=r}^{\infty} E_{k}$ and $V=\bigcap_{k=1}^{\infty} V_{k}$. For each $r \in \mathbb{N}$, we have

$$
\lambda^{n}(E) \leq \lambda^{n}\left(\bigcup_{k=r}^{\infty} E_{k}\right) \leq \sum_{k=r}^{\infty} \lambda^{n}\left(E_{k}\right) \leq 2^{-r+1},
$$

so $\lambda^{n}(E)=0$. Obviously $\lambda^{n}(V)=1$.

Now let $x \in V \backslash E$ be given. Then

$$
x \in \bigcap_{k=r_{x}}^{\infty}\left(V \backslash E_{k}\right)
$$

for some $r_{x} \in \mathbb{N}$. Thus

$$
f_{k, i}(x)=\phi_{i}(x) \quad \forall k \geq r_{x}, 1 \leq i \leq n,
$$

and by (2) we have

$$
\left\|\nabla Q_{k}(x)-f_{k}(x)\right\|<2^{-k} \quad \forall k \geq r_{x} .
$$

Thus (1) yields

Hence we have

$$
\left\|\nabla Q_{k}(x)-\phi(x)\right\|<2^{-k} \quad \forall k \geq r_{x}
$$

$$
\lim _{k \rightarrow \infty} \nabla Q_{k}(x)=\phi(x)
$$

Since $x \in V \backslash E$ was arbitrary and $\lambda^{n}(V \backslash E)=1$, we are done.

As a consequence of (1.2), we obtain an interesting dense subspace of the $F$-space $\mathfrak{M}\left(I^{n}, \mathbb{R}^{n}\right)$ with its metric of coordinatewise convergence in measure. 
(1.3) Corollary. For $f=\left(f_{1}, f_{2}, \ldots, f_{n}\right)$ and $g=\left(g_{1}, g_{2}, \ldots, g_{n}\right)$ in $\mathfrak{M}\left(I^{n}, \mathbb{R}^{n}\right)$ put

$$
\rho(f, g)=\max _{1 \leq j \leq n} \int_{I^{n}} \frac{\left|f_{j}-g_{j}\right|}{1+\left|f_{j}-g_{j}\right|} d \lambda^{n} .
$$

Then $\rho$ is a complete invariant metric for $\mathfrak{M}\left(I^{n}, \mathbb{R}^{n}\right)$ and the linear subspace $\left\{\nabla F: F \in C\left(I^{n}\right)\right.$ and $\nabla F$ exists and is continuous on an open set $V \subset\left(I^{n}\right)^{\circ}$ with $\left.\lambda^{n}(V)=1\right\}$ is dense in this space.

Proof. The completeness of $\rho$ is immediate from the well-known special case $n=1$ in which $\rho$-convergence is equivalent to convergence in measure (see [3, p. 93, Theorem E] for completeness). Since a.e. convergence on a finite measure space implies convergence in measure (see $[4,(11.31)]$ ), we need only apply (1.2).

\section{Density in $C\left(I^{n}\right)$ OF APPROXIMATE ANTIGRADIENTS}

In this section we present our main theorem as stated in the abstract above. It improves Theorem (1.1) in that it shows that, for a given $P$ and $\varepsilon$, the set of $Q$ 's that satisfy the conclusion of $(1.1)$ is dense in $C\left(I^{n}\right)$. We need the results of this section for our work on universal primitives that is in preparation.

We begin with three lemmas.

(2.1) Lemma. Let $n \in \mathbb{N}$ be given and let $\{0,1\}^{n}=\left\{u \in \mathbb{R}^{n}: u_{j} \in\{0,1\}\right.$, $j=1,2, \ldots, n\}$. Suppose that $y_{u} \in \mathbb{R}$ is given $\forall u \in\{0,1\}^{n}$, and let $m$ be the minimum and $M$ the maximum of $\left\{y_{u}: u \in\{0,1\}^{n}\right\}$. Then there exist a function $f \in C\left(I^{n}\right)$ and an open set $V \subset\left(I^{n}\right)^{\circ}$ with $\lambda^{n}(V)=1$ such that

(i) $\nabla f(x)=0 \quad \forall x \in V$,

(ii) $f(u)=y_{u} \forall \dot{u} \in\{0,1\}^{n}$, and

(iii) $m \leq f(x) \leq M \quad \forall x \in I^{n}$.

Proof. If $n=1$, let $f$ be defined by

$$
f(x)=y_{0}+\left(y_{1}-y_{0}\right) \cdot \psi(x) \quad \forall x \in I=[0,1],
$$

and take $V=I \backslash C$, where $\psi$ is Lebesgue's singular function and $C$ is Cantor's ternary set. Then (i)-(iii) are obvious.

Suppose, as an induction hypothesis, that $n>1$ and the theorem is true if $n$ is replaced with $n-1$.

For $l \in\{0,1\}$, we identify the slice

$$
S_{l}=\left\{x \in I^{n}: x_{n}=l\right\}
$$

with $I^{n-1}$ in the obvious way and write

$$
\begin{aligned}
& S_{l}^{\circ}=\left\{x \in S_{l}: 0<x_{j}<1,1 \leq j \leq n-1\right\}, \\
& A_{l}=\left\{u \in\{0,1\}^{n}: u_{n}=l\right\} .
\end{aligned}
$$

By the induction hypothesis, we obtain an $f_{l} \in C\left(S_{l}\right)$ and an open set $V_{l} \subset S_{l}^{\circ}$ with $\lambda^{n-1}\left(V_{l}\right)=1 \quad(l=0,1)$ such that

$$
\begin{gathered}
\frac{\partial f_{l}}{\partial x_{j}}(x)=0 \quad \forall x \in V_{l}, \quad 1 \leq j<n, \\
f_{l}(u)=y_{u} \quad \forall u \in A_{l} \quad \text { and } \quad m \leq f_{l}(x) \leq M \quad \forall x \in S_{l} .
\end{gathered}
$$


For any $x=\left(x_{1}, x_{2}, \ldots, x_{n}\right) \in I^{n}$, define $x^{l} \in S_{l}$ by $\left(x^{l}\right)_{j}=x_{j}$ if $j<n$ and $\left(x^{l}\right)_{n}=l$ for $l=0,1$. Define $f: I^{n} \rightarrow \mathbb{R}$ by

$$
\begin{aligned}
f(x) & =f_{0}\left(x^{0}\right)+\left[f_{1}\left(x^{1}\right)-f_{0}\left(x^{0}\right)\right] \cdot \psi\left(x_{n}\right) \\
& =\left[1-\psi\left(x_{n}\right)\right] \cdot f_{0}\left(x^{0}\right)+\psi\left(x_{n}\right) \cdot f_{1}\left(x^{1}\right) .
\end{aligned}
$$

Also define

$$
V=\left(I^{n}\right)^{\circ} \cap\left(\bigcap_{l=0}^{1}\left\{x \in I^{n}: x^{l} \in V_{l}\right\}\right) \cap\left(\left\{x \in I^{n}: x_{n} \notin C\right\}\right) .
$$

It is obvious that $V$ is open and $\lambda^{n}(V)=1$. The continuity of the functions $x \rightarrow x^{l} \quad(l=0,1)$, each $f_{l}$, and $\psi$ ensures that $f$ is continuous on $I^{n}$. Plainly, $\nabla f(x)=0 \forall x \in V$ by the definition of $f$ and the definition of $V$. This proves (i).

If $u \in A_{0}$, then $u_{n}=0, u=u^{0}$, and $\psi\left(u_{n}\right)=0$, hence $f(u)=f_{0}(u)=y_{u}$. If $u \in A_{1}$, then $u_{n}=1, u=u^{1}$, and $f(u)=f_{1}(u)=y_{u}$. Thus (ii) holds.

Since $0 \leq \psi \leq 1, f(x)$ is between $f_{0}\left(x^{0}\right)$ and $f_{1}\left(x^{1}\right)$ so (iii) holds too.

(2.2) Remark. It is not difficult to replace $I^{n}$ with any closed interval

$$
\Omega=[a, b]=\left\{x \in \mathbb{R}^{n}: a_{j} \leq x_{j} \leq b_{j}, 1 \leq j \leq n\right\},
$$

where $a=\left(a_{1}, a_{2}, \ldots, a_{n}\right)$ and $b=\left(b_{1}, b_{2}, \ldots, b_{n}\right)$.

(2.3) Lemma. Let $n \in \mathbb{N}$, let $G \in C\left(I^{n}\right)$, and let $\varepsilon>0$. Then there exist $a$ function $H \in C\left(I^{n}\right)$ and an open set $V \subset\left(I^{n}\right)^{\circ}$ with $\lambda^{n}(V)=1$ such that

(i) $\nabla H(x)=0 \forall x \in V$,

(ii) $\|G-H\|_{u}<\varepsilon$,

where $\|F\|_{u}=\sup \left\{|F(x)|: x \in I^{n}\right\}$ for any $F \in C\left(I^{n}\right)$.

Proof. Suppose $n=1$. Find $N \in \mathbb{N}$ such that $|G(u)-G(v)|<\varepsilon / 2$ if $u, v \in I$ and $|u-v| \leq 1 / N$. As before, let $C$ be Cantor's ternary set and let $\psi$ be Lebesgue's singular function. Define

$$
H(x)=G\left(\frac{k}{N}\right)+\left[G\left(\frac{k+1}{N}\right)-G\left(\frac{k}{N}\right)\right] \cdot \psi(N x-k)
$$

if $k \in\{0,1, \ldots, N-1\}$ and $\frac{k}{N} \leq x \leq \frac{k+1}{N}$, also define

$$
V=\bigcup_{k=0}^{N-1}\{x \in I: k \leq N x \leq k+1,(N x-k) \notin C\} .
$$

Notice that $H\left(\frac{k}{N}\right)=G\left(\frac{k}{N}\right)$ for $k=0,1, \ldots, N, H \in C(I), \lambda(V)=1$, and $k \leq N x \leq k+1 \Rightarrow$

$$
\begin{aligned}
|H(x)-G(x)| & \leq\left|H(x)-G\left(\frac{k}{N}\right)\right|+\left|G\left(\frac{k}{N}\right)-G(x)\right| \\
& <\left|G\left(\frac{k+1}{N}\right)-G\left(\frac{k}{N}\right)\right| \cdot \psi(N x-k)+\frac{\varepsilon}{2}<\varepsilon .
\end{aligned}
$$

Plainly, $x \in V \Rightarrow \nabla H(x)=H^{\prime}(x)=0$ so the lemma holds for $n=1$.

Suppose $n>1$ and, as an induction hypothesis, that the lemma is true if $n$ is replaced by $n-1$. The uniform continuity of $G$ provides $\delta>0$ such that

$$
u, v \in I^{n},\|u-v\|<\delta \Rightarrow\|G(u)-G(v)\|<\varepsilon / 8 \text {. }
$$


Choose $N \in \mathbb{N}$ with $N \delta>1$. For any $r \in\{0,1, \ldots, N\}$, we identify the slice

$$
S_{r}=\left\{x \in I^{n}: x_{n}=r / n\right\}
$$

with $I^{n-1}$ in the obvious way, and we write

$$
S_{r}^{\circ}=\left\{x \in S_{r}: 0<x_{j}<1 \forall j<n\right\} .
$$

For each such $r$, we apply our induction hypothesis to the restriction of $G$ to $S_{r}$ to obtain a continuous function $G_{r} \in C\left(S_{r}\right)$ and an open set $V_{r} \subset S_{r}^{\circ}$ which is open in $S_{r}$ such that

$$
\begin{aligned}
& \lambda^{n-1}\left(V_{r}\right)=1, \\
& \frac{\partial G_{r}}{\partial x_{j}}(x)=0 \quad \forall x \in V_{r}(1 \leq j<n), \text { and } \\
& \left|G(x)-G_{r}(x)\right|<\frac{\varepsilon}{8} \quad \forall x \in S_{r} .
\end{aligned}
$$

Next we construct $H$ on $I^{n}$ which extends each $G_{r}$ and satisfies the requirements of the lemma.

The construction of $H$ is exactly the same as that of $Q_{n}$ in the proof of Theorem (1.1). That is,

$$
H(x)=G_{r-1}\left(x^{r-1}\right)+\left[G_{r}\left(x^{r}\right)-G_{r-1}\left(x^{r-1}\right)\right] \cdot \psi\left(N x_{n}-r+1\right)
$$

if $(r-1) / N \leq x_{n} \leq r / N$. Also define

$$
\begin{aligned}
V= & \left(I^{n}\right)^{\circ} \cap\left(\bigcap_{r=0}^{N}\left\{x \in I^{n}: x^{r} \in V_{r}\right\}\right) \\
& \cap\left(\bigcup_{r=1}^{N}\left\{x \in I^{n}: r-1 \leq N x_{n} \leq r,\left(N x_{n}-r+1\right) \notin C\right\}\right),
\end{aligned}
$$

where $C$ is Cantors ternary set. Plainly $V$ is open, $\lambda^{n}(V)=1, H \in C\left(I^{n}\right)$, and $\nabla H(x)=0 \quad \forall x \in V$.

Now let $x \in V$ with $r-1<N x_{n}<r$. Then we have

$$
\left\|x-x^{r}\right\|<\left\|x^{r-1}-x^{r}\right\|=1 / N<\delta \text { and also }\left\|x-x^{r-1}\right\|<\delta .
$$

From (1) and (2) we have

$$
\begin{aligned}
|H(x)-G(x)|= & \left|G_{r-1}\left(x^{r-1}\right)+\left[G_{r}\left(x^{r}\right)-G_{r-1}\left(x^{r-1}\right)\right] \cdot \psi\left(N x_{n}-r+1\right)-G(x)\right| \\
\leq & \left|G_{r-1}\left(x^{r-1}\right)-G\left(x^{r-1}\right)\right|+\left|G\left(x^{r-1}\right)-G(x)\right| \\
& +\left|G_{r}\left(x^{r}\right)-G_{r-1}\left(x^{r-1}\right)\right| \cdot\left|\psi\left(N x_{n}-r+1\right)\right| \\
\leq & \frac{\varepsilon}{8}+\frac{\varepsilon}{8}+\left|G_{r}\left(x^{r}\right)-G_{r-1}\left(x^{r-1}\right)\right| \\
\leq & \frac{\varepsilon}{4}+\left|G_{r}\left(x^{r}\right)-G\left(x^{r}\right)\right|+\left|G\left(x^{r}\right)-G\left(x^{r-1}\right)\right| \\
& +\left|G\left(x^{r-1}\right)-G_{r-1}\left(x^{r-1}\right)\right| \\
\leq & \frac{\varepsilon}{4}+\frac{\varepsilon}{8}+\frac{\varepsilon}{8}+\frac{\varepsilon}{8}<\varepsilon .
\end{aligned}
$$

Thus $\|H-G\|_{u}<\varepsilon$ on $I^{n}$. This completes the proof.

(2.4) Lemma. Let $n \in \mathbb{N}$ and let $Q \in C\left(I^{n}\right)$ be such that there exists an open set $V \subset\left(I^{n}\right)^{\circ}$ with $\lambda^{n}(V)=1$ on which $\nabla Q$ exists and is continuous. Suppose 
that $F_{0} \in C\left(I^{n}\right)$ and $\varepsilon>0$. Then there exist $F \in C\left(I^{n}\right)$ and an open set $W \subset V$ with $\lambda^{n}(W)=1$ such that

(i) $\nabla F$ exists and is continuous on $W$,

(ii) $\nabla F(x)=\nabla Q(x) \quad \forall x \in W$, and

(iii) $\left\|F-F_{0}\right\|_{u}<\varepsilon$.

Proof. Apply Lemma (2.3) to $G=F_{0}-Q$ to obtain $H \in C\left(I^{n}\right)$ and an open set $V_{0} \subset\left(I^{n}\right)^{\circ}$ with $\lambda^{n}\left(V_{0}\right)=1$ such that $\|H-G\|_{u}<\varepsilon$ and $\nabla H(x)=0$ $\forall x \in V_{0}$. Take $F=Q+H$ and $W=V \cap V_{0}$ to complete the proof.

(2.5) Main Theorem. Let $n \in \mathbb{N}$, let $f \in C\left(I^{n}, \mathbb{R}^{n}\right)$, let $F_{0} \in C\left(I^{n}\right)$, and let $\varepsilon>0$. Then there exist $F \in C\left(I^{n}\right)$ and an open set $W \subset\left(I^{n}\right)^{\circ}$ with $\lambda^{n}(W)=1$ such that

(i) $\nabla F$ exists and is continuous on $W$,

(ii) $\|\nabla F(x)-f(x)\|<\varepsilon \quad \forall x \in W$, and

(iii) $\left|F-F_{0}\right|_{u}<\varepsilon$,

where $\|y\|=\left(\sum_{j=1}^{n} y_{j}^{2}\right)^{1 / 2} \quad \forall y \in \mathbb{R}^{n}$.

Proof. Take $P=f$ in (1.1) to produce a $Q$ and then use (2.4) to replace $Q$ with $F$.

\section{REFERENCES}

1. Stanislaw Saks, Theory of the integral, Stechert, New York, 1937.

2. Karl R. Stromberg, An introduction to classical real analysis, Wadsworth, Belmont, CA, 1981.

3. Paul R. Halmos, Measure theory, Van Nostrand, New York, 1950.

4. Edwin Hewitt and Karl Stromberg, Real and abstract analysis, Springer-Verlag, New York, 1965.

Department of Mathematics, Morgan State University, Baltimore, Maryland 21239

E-mail address: GANQMOENG2.MORGAN.EDU

Department of Mathematics, Kansas State University, Manhatten, Kansas 66506 